\title{
Plasma Focused Ion Beam Curtaining Artifact Correction by Fourier-Based Linear Opti-mization Model
}

\author{
Christopher W. Schankula ${ }^{1}$, Christopher K. Anand ${ }^{1}$ and Nabil D. Bassim ${ }^{2}$
}

1. Department of Computing and Software (CAS), McMaster University, Hamilton, Ontario, Canada

2. Department of Materials Science and Engineering (MSE), McMaster University, Hamilton, Ontario, Canada

Focused ion beam scanning electron microscope tomography is a destructive slice-and-image technique for obtaining three-dimensional structural information. Xenon plasma focused ion beam (PFIB) is a promising new ion source technology that has a much higher material removal rate than traditional gallium source technology — greatly increasing the analyzable volume of material [1].

Unfortunately, due to the heterogeneous nature of any interesting sample, plasma milling rates vary, causing vertical ripples ("curtains") on the imaged cut face of the sample. These curtains appear as dark or light straight-line artifacts on secondary and backscatter electron images. In order to perform an accurate automated quantitative analysis of the resulting tomogram, these artifacts must be reduced as much as possible through physical means, such as using a deposited capping layer on the top of the sample or through a "rocking polish" technique which works by milling the sample at two discrete angles between image acquisitions. While these techniques help to reduce the ef-fect, curtains remain at two angles and need to be further reduced through image processing means.

We have developed a new algorithm to effectively correct these image artifacts by applying a Fourier-based linear optimization model to detect and remove curtains at two discretized angles. The algorithm and linear optimization model was implemented in the Anaconda Python 3.5.2 distribution using the open-source Gnu Linear Programming Kit (GLPK) to solve the linear optimization problem on small image blocks, then stitch the corrected image back together.

In order to test the algorithm, we ran the algorithm on a ultra-high performance concrete tomogram taken on an FEI PFIB. A backscatter electron detector was used to take images at $48 \mathrm{~nm} \times 48 \mathrm{~nm}$ pixel resolution with a 50nm slice thickness. During imaging, a rocking mill was used, leaving curtains at approximately $1^{\circ}$ and $-7^{\circ}$ from the vertical. We have shown that our algorithm is able to effectively detect and correct curtaining artifacts without causing changes to other structures (see figures $1 \& 2$ ).

Future work includes taking advantage of the parallel nature of the algorithm and using faster implementations and solvers in order to speed up the processing. Additionally, by combining multispectral data, such as information from both backscatter and secondary electron images, we believe we can obtain more effective curtain detection and correction. Other works meant to reduce only vertical curtains (such as [2]) take advantage of three-dimensional inter-slice information, which may prove advantageous to our model and should be further explored [3]. 
References:

[1] T Burnett et al., Ultramicroscopy 161 (2016), p. 119-129

[2] JH Fitschen, J Ma and S Schuff, Computer Vision and Image Understanding 155 (2017), p.

24-32

[3] The authors acknowledge funding from NSERC and McMaster's MSE department. We thank Prof. Kay Wille for preparing the UHPC sample and Ron Kelley \& Brandon Van Leer of FEI-Thermo Fisher for the imaging.

Original Image

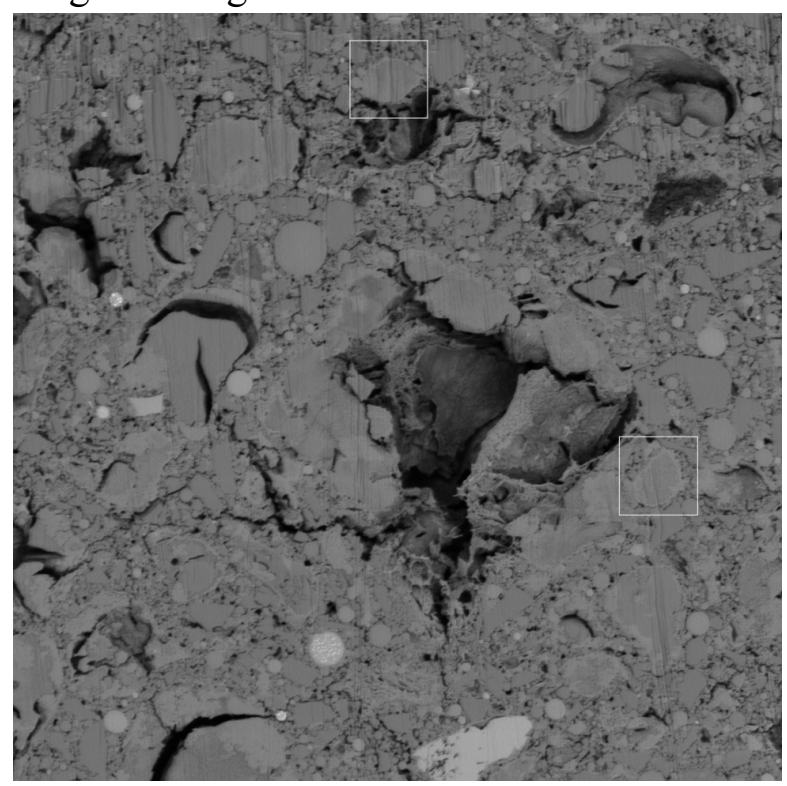

\section{Corrected Image}

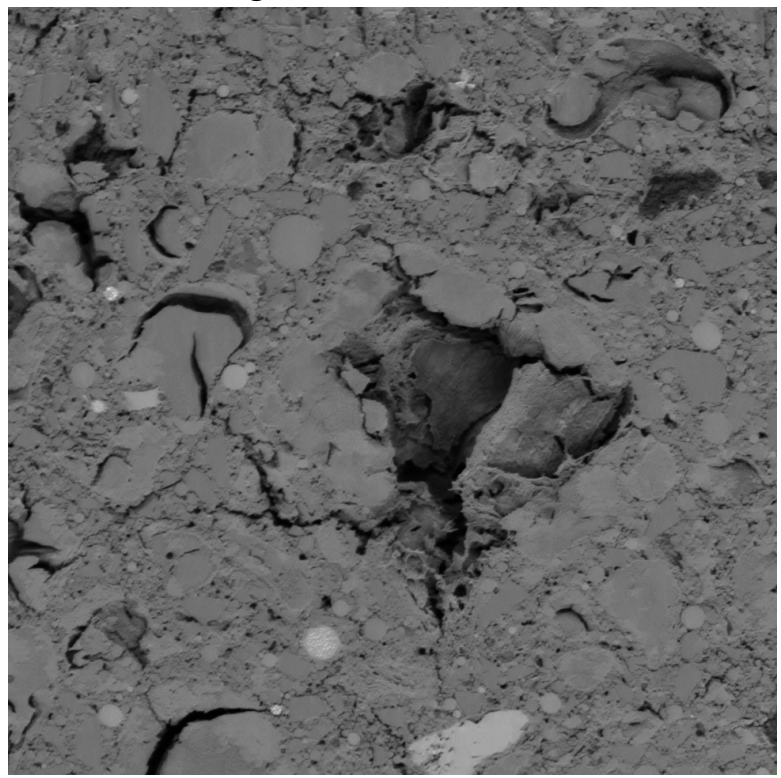

Figure 1. Comparison shows effective correction of curtaining structures in image. Highlighted sections in the original image are blown up in figure 2.

a) Original

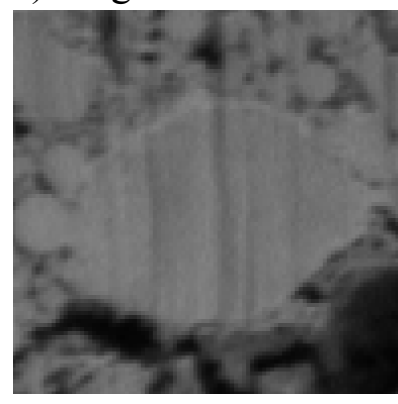

a) Corrected

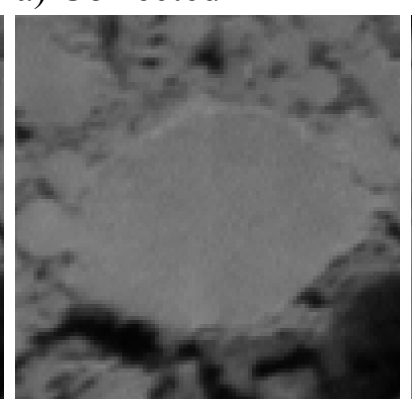

b) Original

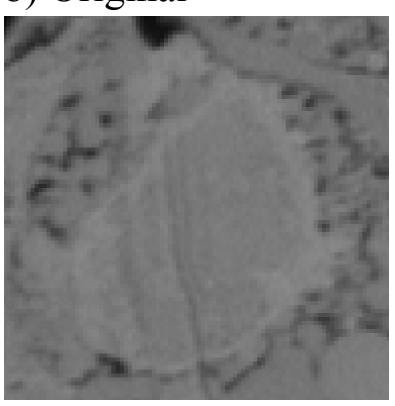

b) Corrected

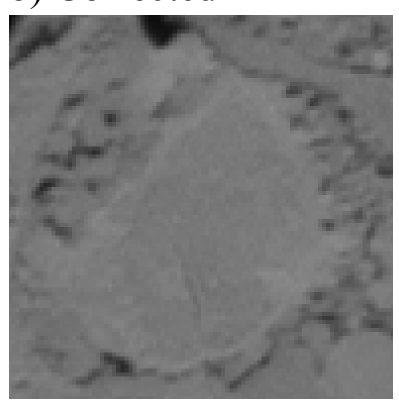

Figure 2. A comparison of blown up sections shown in figure 1. In a), the particle is highly corrupted by curtains in the original image. In b), a small amount of curtaining is left due to a slight curtain deviation, showing that our model targets only specific angles. 